\title{
Negative Ion-Driven Associated Particle Neutron Generator
}

\author{
A.J. Antolak, K-.N. Leung ${ }^{\mathrm{a}}$, D.H. Morse, D.C. Donovan ${ }^{\mathrm{b}}$, \\ J.M. Chames, J.A. Whaley, D.A. Buchenauer, A.X. Chen $^{\mathrm{c}}$ \\ Sandia National Laboratories, Livermore CA 94550 \\ P.A. Hausladen, F. Liang \\ Oak Ridge National Laboratory, Oak Ridge, TN 37831
}

\begin{abstract}
An associated particle neutron generator is described that employs a negative ion source to produce high neutron flux from a small source size. Negative ions produced in an rf-driven plasma source are extracted through a small aperture to form a beam which bombards a positively biased, high voltage target electrode. Electrons co-extracted with the negative ions are removed by a permanent magnet electron filter. The use of negative ions enables high neutron output (100\% atomic ion beam), high quality imaging (small neutron source size), and reliable operation (no high voltage breakdowns). The neutron generator can operate in either pulsed or continuous-wave $(\mathrm{cw})$ mode and has been demonstrated to produce $10^{6} \mathrm{D}-\mathrm{D} \mathrm{n} / \mathrm{s}$ (equivalent to $\sim 10^{8} \mathrm{D}-\mathrm{T} \mathrm{n} / \mathrm{s}$ ) from a 1-mm-diameter neutron source size to facilitate high fidelity associated particle imaging.

\section{Introduction}

Neutron interrogation using the associated particle imaging technique provides element-specific information for detecting and characterizing concealed materials (e.g., nuclear materials, explosives, drugs, and other contraband) in two- or three-dimensions. The technique is based on the deuterium-tritium (D-T) fusion reaction which produces a $14 \mathrm{MeV}$ neutron and an associated 3.5 MeV alpha particle traveling in opposite directions. A particle detector located inside the neutron generator detects the alphas whose trajectories define a solid angle cone beam of neutrons that are used to interrogate an object. When the neutron beam interacts with nuclei in the interrogated object, it stimulates characteristic gamma-ray emission from inelastic scattering events. Coincidence measurements of the gamma signals with the alpha particles provide information on where the inelastic (n, $\gamma$ ) scattering events occurred, while their energy spectra provides compositional information of the region probed in the object. Due to the isotropic nature of the produced gamma signals, detectors can be placed on the same side of the interrogated object as the neutron source or where ever it is convenient for the specific interrogation scenario. The associated particle technique significantly reduces background noise in the measurements since unassociated neutron-induced signals emitted from surrounding regions are disregarded, thereby significantly improving the signal-to-noise ratio.
\end{abstract}

Compact associated particle-type neutron generators in use today accelerate a positive ion beam to about $100 \mathrm{keV}$ which then bombards a target and produces neutrons via the D-T fusion

\footnotetext{
${ }^{a}$ Also affiliated with Department of Nuclear Engineering, University of California, Berkeley, CA.

${ }^{\mathrm{b}}$ Present address: Department of Nuclear Engineering, University of Tennessee, Knoxville, TN.

${ }^{\mathrm{c}}$ Present address: Adelphi Technology, Inc., Redwood City, CA.
} 
reaction. Most systems extract the beam from a Penning discharge source, although other ion sources have been explored. ${ }^{1-5}$ Penning discharges are magnetically supported gas discharges at relatively low pressures. Once plasma has been ignited, ionizing electrons are emitted from a cold cathode due to ion bombardment. A superimposed magnetic field forces the electrons to follow a cycloidal path. Because of the low pressure in the discharge chamber, the mean free path length of the electrons is long enough to allow them to gain the energy needed to efficiently ionize the operating gas. Penning sources primarily produce molecular ions (>90\%), so both the ion current and energy are generally increased to achieve higher neutron flux from the target. ${ }^{4}$ The beam extraction aperture is located at the anti-cathode electrode which can become degraded or enlarged due to sputtering which causes the neutron source size to grow over time and leads to progressively poorer imaging resolution. When the ion beam bombards the target, it produces secondary emission electrons. As these electrons stream back to the ion source, they will form part of the current drain in the high voltage power supply. In this case, the target current is effectively greater (by an unknown amount) than the incident beam current and this ambiguity makes measuring and controlling the beam current very difficult. The backstreaming electrons can also cause damage on the ion source or the accelerator column if they are not suppressed at the target electrode. To mitigate these effects, a negatively biased shroud electrode is sometimes placed near or around the target, but sparking and detector damage in the neutron generator can still occur. ${ }^{6}$

In this new negative ion-driven associated particle neutron generator (Fig. 1), the target is biased at $+100 \mathrm{kV}$ relative to ground potential. A beam of negative deuterium $\left(\mathrm{D}^{-}\right)$ions is extracted from a volume production-type negative ion source which is maintained at ground potential. Unlike the case of a negative biased target, the electrons emitted from the target electrode will be attracted back and the issues associated with backstreaming electrons as well as high voltage breakdown are completely eliminated. The accelerated ion beam contains $100 \%$ pure atomic $\mathrm{D}^{-}$ ions which provide full energy D-D fusion reactions to occur at the beam-loaded target and result in enhanced neutron yield.

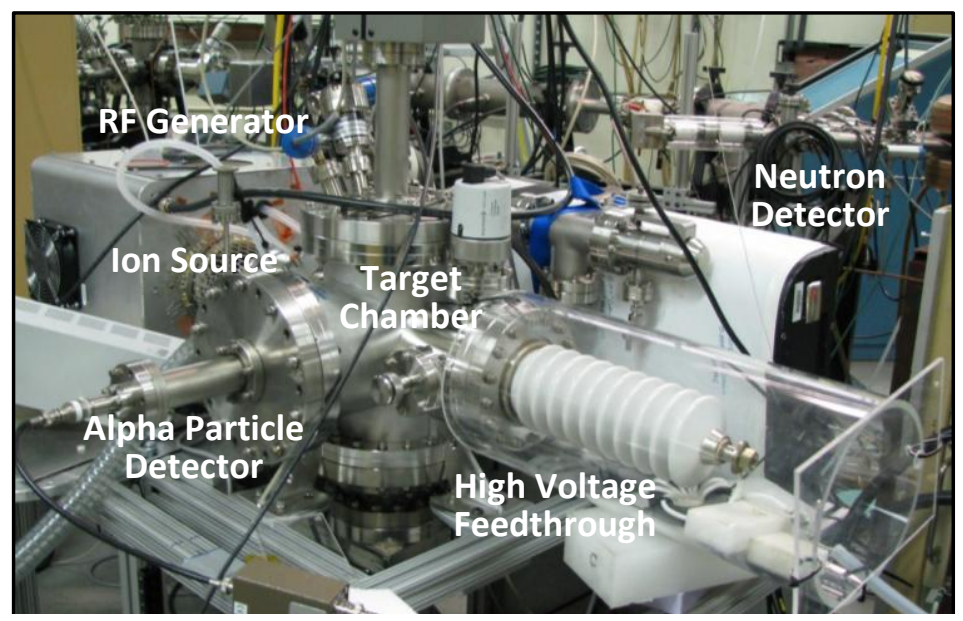

Figure 1. Photo of the negative ion-driven associated particle neutron generator.

\section{Neutron Generator}

The problems encountered in neutron generators when using positive ions can be mitigated or even eliminated by employing negative ions. Among the different techniques for producing 
negative ions, direct extraction from a volume production-type gas discharge has several advantages. The negative ions generated by volume-production processes have lower beam emittance and thus are useful for the generation of high brightness beams. Additionally, a volume-production source can provide sufficient amount of $\mathrm{D}^{-}$ions for the associated particle technique without the use of cesium which greatly simplifies the construction and operation of the ion source. ${ }^{7}$ In the volume-production ion source, negative deuterium ion formation is based on the greatly increased cross section for the dissociative-attachment reaction when molecules are excited to high vibrational states. During collisions with walls or energetic $\left(T_{e}>5 \mathrm{eV}\right)$ electrons, deuterium molecules are excited to high vibrational states and the cross-section for the dissociative attachment reaction $\left(T_{e}<1 \mathrm{eV}\right)$ increases by orders-of-magnitude for high excited states. $^{8}$ While competing destructive reactions (neutralization, associative detachment, and electronic detachment) can be important, the increased production due to high vibrational states results in a significant negative ion population in the plasma. The lack of filaments, simplicity of design, easy maintenance and long lifetime are the well-known advantages of radio frequency (RF) sources compared with arc discharge sources. Previous work has shown that negative hydrogen $\left(\mathrm{H}^{-}\right)$ion current density of $\sim 10 \mathrm{~mA} / \mathrm{cm}^{2}$ can be achieved with $1 \mathrm{~kW}$ of input RF-power at $2 \mathrm{MHz}$ frequency. ${ }^{9}$ These current densities were achieved by optimizing the extraction chamber length and by adding very low energy electrons into the extraction region. In the present associated particle neutron generator, yields of $10^{6} \mathrm{D}-\mathrm{D} \mathrm{n} / \mathrm{s}$ have been demonstrated from a 1-mm-diameter beam spot with $\sim 600 \mu \mathrm{A}, 100 \mathrm{kV}$ negative ion beam on target. A plasma current density $\left(\sim 35 \mathrm{~mA} / \mathrm{cm}^{2}\right)$ was extracted from the ion source with $\sim 1 \mathrm{~kW}$ of RF-discharge power at $13.56 \mathrm{MHz}$ frequency and by using pure gas discharge. The ion source and target chambers are differentially pumped to prevent voltage breakdown and minimize charge neutralization in the acceleration column.

The ion source chamber in the neutron generator is a 8-cm-diameter by 5-cm-long quartz cup with a flat spiral "pancake" copper antenna externally located on the closed end (see Fig. 2). The plasma is produced by $13.56 \mathrm{MHz}$ RF induction discharge generated by the antenna coil. Quartz was selected for the source chamber because it provides excellent RF-coupling to the plasma, can be hard sealed to stainless steel, forms a good hydrogen isotope barrier, and retains structural robustness at high temperatures. A pair of water cooled permanent magnet filter rods are installed on the exit flange of the source chamber. The magnetic filter divides the source chamber into a discharge region and an extraction region by providing a narrow region of transverse magnetic field which is strong enough ( 270 Gauss at the center) to prevent the energetic electrons from entering the extraction zone. A stainless-steel mesh is also mounted in front of the magnetic filter to provide filtering of the RF-field inside the extraction region and improved survivability of negative ions. Excitation and ionization of the gas molecules by energetic electrons occurs in the discharge region, while plasma composed of positive and negative ions as well as cold electrons are found in the extraction region.

In normal operation, a negative ion beam is extracted from the plasma source through a small $(1.5$-mm-diameter) aperture at low voltage $(\sim 5 \mathrm{kV})$. Electrons in the beam are removed by a cross B-field while the negative ions pass through essentially un-deflected and then are accelerated to a positively biased $(+100 \mathrm{kV})$ target electrode where neutron-producing fusion reactions occur on the surface. Although the actual pressure in the ion source could not be measured, the nominal feed gas pressure into the ion source is kept at approximately 75 mTorr. 
Differential pumping is used to keep the pressure in the target chamber at around $0.01 \mathrm{mTorr}$ and the extraction aperture-to-target distance is about $6 \mathrm{~cm}$. The cross section for neutralizing negative hydrogen $\left(\mathrm{H}^{-}\right)$ions from interactions with ambient $\mathrm{H}_{2}$ gas is $\sim 10^{-15} \mathrm{~cm}^{2}$ over the energy range of $10 \mathrm{eV}$ to $100 \mathrm{keV} .{ }^{10}$ Analogously, the cross section for neutralizing negative deuterium ions is expected to be about the same value over this energy range. In this case, the mean free path for charge neutralization in the target chamber will be $\sim 30 \mathrm{~m}$. The beam extracted from the source also contains a substantial amount of electrons which need to be removed before they are accelerated to high energy. This is accomplished by using a small permanent magnet electron filter (see Fig. 2). A field of $\sim 1.5$ kilo-Gauss at the center of the filter is optimal for sweeping out the extracted electrons while allowing the $\mathrm{D}^{-}$ions to pass through with very little deflection. The electron filter is lined with thin carbon plates containing small grooves to trap the reflected electrons and prevent them from "bouncing" out of the filter.

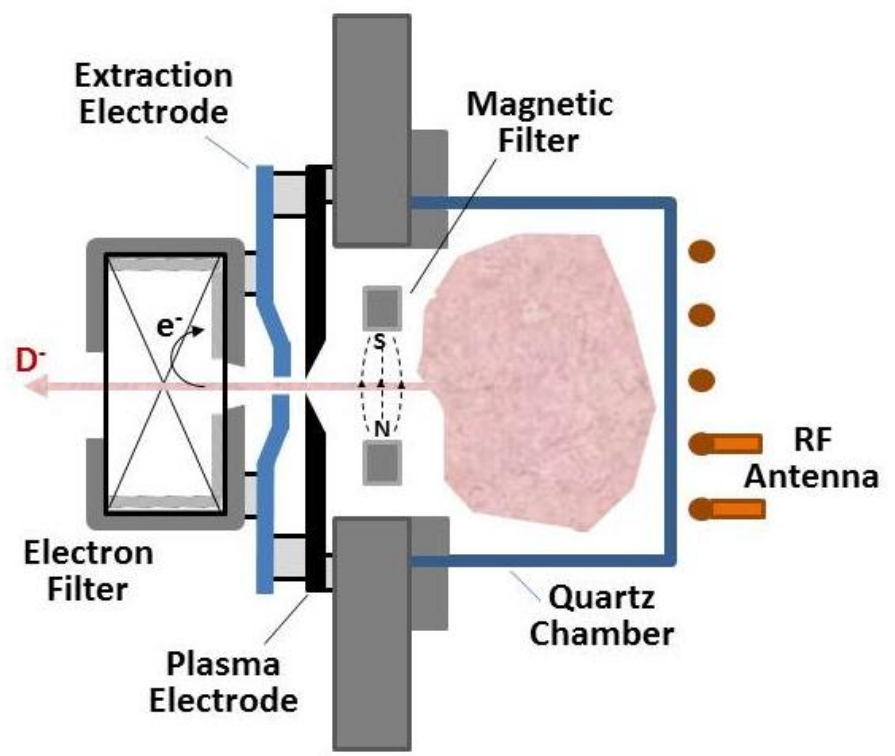

Figure 2. Schematic diagram of the rf-driven plasma negative ion source and permanent magnet electron filter.

Upon exiting the electron filter, the negative ion beam is accelerated to the positively biased, high voltage $(+100 \mathrm{kV})$ target electrode. High voltage to the target is supplied through an $8-\mathrm{mm}-$ diameter copper rod that is installed parallel to the ion beam axis and also acts as a heat sink for the target. The target consists of an 8-mm-diameter $\mathrm{x} 1$-mm-thick titanium disc that is attached to the $45^{\circ}$-beveled end of the copper rod terminal. Titanium is used as the target material because it can adsorb deuterium and tritium efficiently on its surface. As the beam loads the target, D-D fusion reactions occur on the surface producing $2.45-\mathrm{MeV}$ neutrons and associated $0.82-\mathrm{MeV}^{3} \mathrm{He}$-particles that travel in opposite directions along the same spatial vector. The beveled end of the target faces the particle detector so the produced ${ }^{3} \mathrm{He}$-particles have a direct path to the detector. The neutrons, which exit in the opposite direction, pass through the titanium target and copper terminal rod. Since the total thickness of traversed material is very small $(<5$ $\mathrm{mm}$ ) only a negligible amount of scattering occurs. Little or no high voltage breakdowns occur inside the neutron generator since secondary electrons emitted from ion bombardment cannot escape the positively biased target. 
The alpha detector is mounted inside the neutron generator so it must be compatible with vacuum and cannot outgas. The detector currently implemented in the neutron generator is a 50 mm diameter $x 0.1 \mathrm{~mm}$ thick YAP:Ce scintillator. The surface of the scintillator is covered with a $\sim 1-\mu \mathrm{m}$-thick aluminum film to prevent scattered or sputtered ions from reaching the detector. The coating also stops low-energy alpha particles thus reducing the number of neutrons tagged by the generator. These lower energy alphas originate from the fusion reactions that occurred below the target surface and have lost energy from multiple scattering. The aluminum coating is mechanically held in place on a Schott type 75C fiber-optic faceplate. The YAP:Ce crystal is located $\sim 6 \mathrm{~cm}$ from the neutron production target giving a field-of-view with an opening angle of roughly 47 degrees. The alpha particle detector is viewed through the fiber optic faceplate and a pixelated light guide by a Hamamatsu H9500 256-anode photomultiplier tube that is electronically divided into 4 quadrants, each with 4 -corner readout. The pixelated light guide serves to linearize and discretize the position response of the photomultiplier tube to give 1-mm pixel detector resolution matching the 1-mm-diameter neutron source size.

\section{Experimental Results}

High fidelity associated particle imaging requires high neutron output, small neutron source size, and reliable operation. The operational characteristics of the neutron generator are listed in Table 1. The experimental results in this paper were obtained with pure deuterium as the feed gas.

Table 1. Negative ion-driven associated particle neutron generator operational characteristics.

\begin{tabular}{|c|c|}
\hline RF Power & $<1000 \mathrm{~W}$ \\
\hline Beam Current & $0.6 \mathrm{~mA}$ \\
\hline Beam Energy & $100 \mathrm{kV}$ \\
\hline Beam Spot Size & $\leq 1 \mathrm{~mm}^{2}$ \\
\hline Atomic Ion Fraction & $100 \%$ \\
\hline D-D Neutron Yield & $10^{6} \mathrm{n} / \mathrm{s}$ in $4 \pi$ \\
\hline Lifetime & 1000 hours \\
\hline
\end{tabular}

\section{a. Neutron Yield}

In associated particle neutron generators, the neutron output is typically limited to $\sim 10^{8} \mathrm{D}-\mathrm{T} \mathrm{n} / \mathrm{s}$ (equivalent to $10^{6} \mathrm{D}-\mathrm{D} \mathrm{n} / \mathrm{s}$ ) by accidental coincidences arising from long neutron flight paths in the correlation measurements. For $100 \mathrm{keV}$ deuterons bombarding a thick chemically loaded $\mathrm{TiD}_{2}\left(\mathrm{TiT}_{2}\right)$ target, the measured D-D (D-T) neutron output is $10^{6} \mathrm{n} / \mathrm{s}\left(10^{8} \mathrm{n} / \mathrm{s}\right)$ with $5 \mu \mathrm{A}(1.7$ $\mu \mathrm{A}$ ) beam current. ${ }^{11}$ Similarly, the computed neutron output is $10^{8} \mathrm{n} / \mathrm{s}$ for a mixed $4.5 \mu \mathrm{A}, 100$ $\mathrm{keV}$ beam $\left(50 \%\right.$ deuteron $/ 50 \%$ triton) bombarding a $\mathrm{TiT}_{2}$ thick target. ${ }^{12}$ In the negative iondriven associated particle neutron generator, the neutron output was measured using a ${ }^{3} \mathrm{He}$ detector calibrated to a ${ }^{252} \mathrm{Cf}$ reference source of known activity. Initial results indicated that the neutron generator was only producing $\sim 10^{5} \mathrm{D}-\mathrm{D} \mathrm{n} / \mathrm{s}$, albeit at rather high $\mathrm{RF}$-power $(>1 \mathrm{~kW})$ and beam current on target (few $\mathrm{mA}$ ). For very brief periods, neutron yields as high as $10^{6} \mathrm{D}-\mathrm{D}$ $\mathrm{n} / \mathrm{s}$ could also be achieved under these conditions. To increase the neutron output as well as address the non-ideal operating conditions, several design changes were made to the beam extraction electrode, electron filter, and automated control system. For example, the power dissipated in the electron filter is around $40 \mathrm{~W}$ which could cause the samarium-cobalt permanent magnets to be heated above their maximum operating temperature $\left(250{ }^{\circ} \mathrm{C}\right)$. 
Aluminum nitride standoffs were installed to enhance thermal conduction to the cooled plasma electrode while maintaining electrical isolation for the electron filter. Additionally, the extraction electrode was fabricated from soft iron to provide a magnetic field-free gap between the plasma and extraction electrodes so that the extracted negative ion beam is not deflected before entering the electron filter. As an example, the yield plot in Figure 3 shows that the neutron generator stably produced $>10^{6} \mathrm{D}-\mathrm{D} \mathrm{n} / \mathrm{s}$ for extended periods at the operational conditions of $1 \mathrm{~kW}$ RF-power, $0.01 \mathrm{mTorr}$ target chamber pressure, and $400 \mu \mathrm{A}$ beam current.

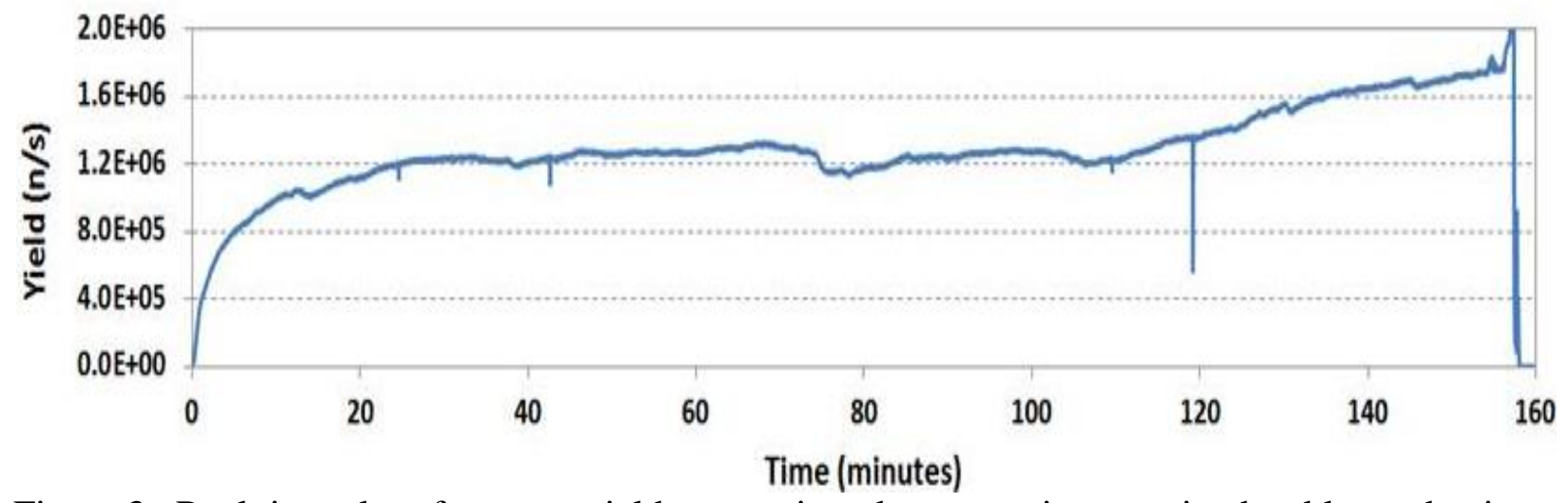

Figure 3. Real time plot of neutron yield versus time demonstrating sustained stable production of $>10^{6} \mathrm{D}-\mathrm{D} \mathrm{n} / \mathrm{s}$ from the prototype negative ion-driven associated particle neutron generator.

\section{a. Neutron Emission Source Size}

Interrogation techniques such as associated particle imaging and radiography benefit from a "point neutron source" to provide high geometrical magnification for imaging. The beam profile was initially analyzed using a three-dimensional particle trajectory computer program with different electrode configurations and target-to-detector orientations. ${ }^{13}$ The computations

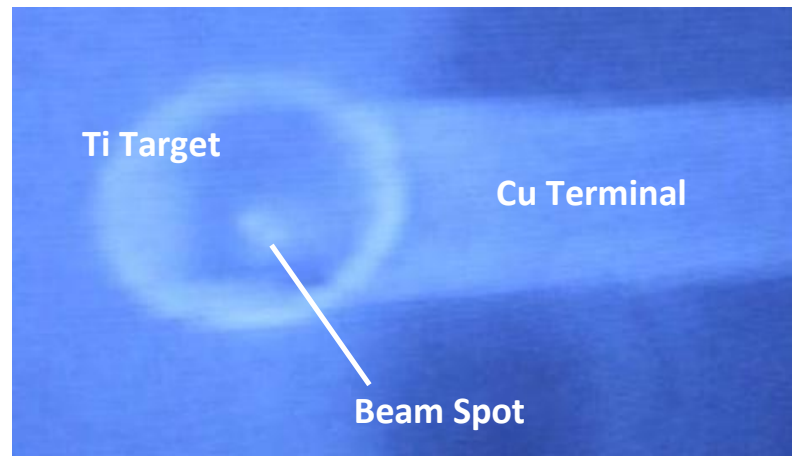

Figure 4. High resolution infrared camera viewing target through chamber viewport. The titanium target diameter is $8 \mathrm{~mm}$ and the ion beam spot size is $\sim 1$-mm-diameter.

predicted the neutron source on the target to be elliptically shaped with dimensions $0.8 \mathrm{~mm} \times 1.1$ $\mathrm{mm}$. The simulations also indicated that the beam strikes the target surface at an angle greater than $45^{\circ}$ due to the field near the target electrode, which helps to minimize the production of sputtered and reflected ions that can damage internal components of the generator. The beam spot size was also measured directly by viewing the target with an externally mounted high- 
resolution infrared camera. Figure 4 shows an infrared camera image of the target. In this case, a positive ion $\left(\mathrm{D}^{+}\right)$beam was used since it was found that the negative $\left(\mathrm{D}^{-}\right)$beam current was too low to get a measurable heat signature. The neutron source size obtained by scaling the imaged beam spot to the known titanium target diameter is approximately 1-mm-diameter.

Table 2. High voltage operational test results.

\begin{tabular}{|c|c|c|c|c|}
\cline { 2 - 5 } \multicolumn{1}{c|}{} & $\begin{array}{c}\text { Feed Gas } \\
\text { Pressure }\end{array}$ & $\begin{array}{c}\text { Target Chamber } \\
\text { Pressure }\end{array}$ & $\begin{array}{c}\text { Positive Ion } \\
\text { Beam }\end{array}$ & $\begin{array}{c}\text { Negative Ion } \\
\text { Beam }\end{array}$ \\
\hline HV without beam & $10^{-4}$ mTorr & $10^{-4}$ mTorr & $-100 \mathrm{kV}$ & $+100 \mathrm{kV}$ \\
\hline HV with beam & 30 mTorr & 0.1 mTorr & $-44 \mathrm{kV}$ & $+100 \mathrm{kV}$ \\
\hline
\end{tabular}

\section{b. High Voltage Operation}

Conventional associated particle-type neutron generators have had reliability issues from high voltage breakdowns that originate from secondary electron emission when the beam hits the target. The electrons are accelerated back to the ion source which is biased at positive high voltage and can cause damage to internal components, produce unwanted x-ray radiation, creat a current drain on the power supply, and cause sparking in the acceleration column. It has been shown that a negatively biased target (for accelerating positive ions) would not cause breakdowns at high voltage and below 10 mTorr operating pressure without an ion beam. ${ }^{4}$ However, in the presence of a positive ion beam the system became unstable at pressures above 5 mTorr and voltage breakdown occurred in the discharge region. Table 2 summarizes the high voltage operational tests performed with the present neutron generator. The results show that stable operation was obtained with the negative ion beam at high voltage $(+100 \mathrm{kV})$ and feed gas pressure of 30 mTorr over several hours. However, when the polarity of the beam was reversed to operate with positive ions, numerous small discharges were observed and stable operation could only be achieved for target voltages up to $-44 \mathrm{kV}$.

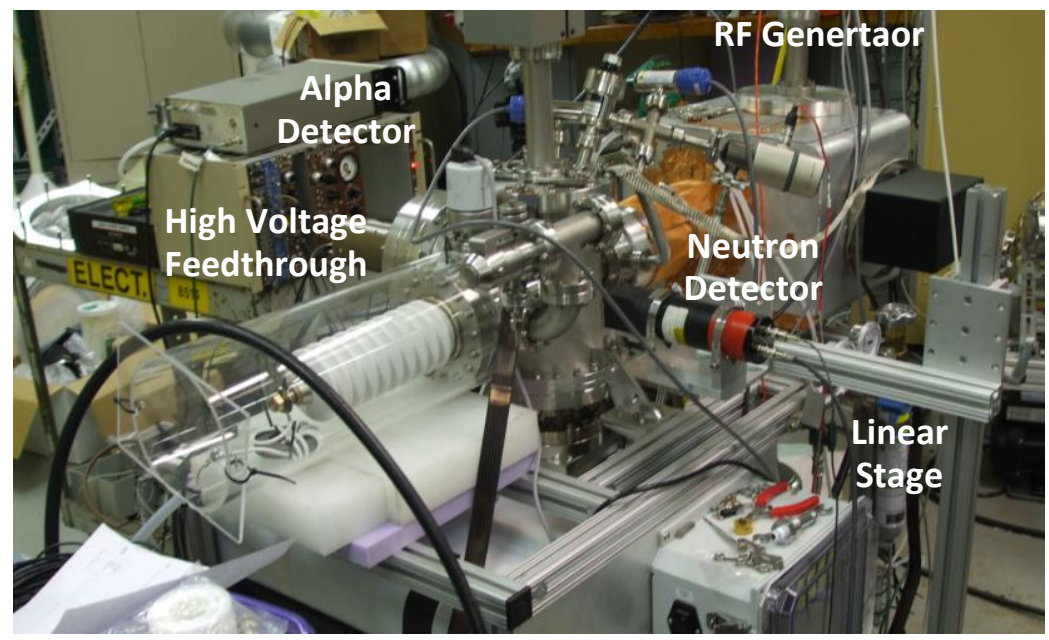

Figure 5. Photograph showing neutron generator with integrated alpha particle detector and the plastic neutron detector mounted on the linear stage for measuring $\mathrm{n}-3 \mathrm{He}$ coincidences.

\section{c. Coincidence Measurements}

In order to demonstrate the associated particle technique with the negative ion-driven neutron generator, experiments were performed to measure coincidences between the $2.45 \mathrm{MeV} \mathrm{D}-\mathrm{D}$ 
neutrons and associated $0.82 \mathrm{MeV}^{3} \mathrm{He}$-particles. The neutrons were detected by a plastic scintillator mounted on a linear translational stage mounted outside the neutron generator while the associated ${ }^{3} \mathrm{He}$-particles were detected by the YAP:Ce scintillator detector inside the generator (Fig. 5). Pulse shape discrimination was used to detect the neutrons since the light generated by neutrons in the plastic scintillator has a longer decay time compared to that produced by $\gamma$-rays. The neutron and ${ }^{3} \mathrm{He}$-particle signals were simultaneously analyzed in two separate channels of a digital oscilloscope. A trace of coincident neutrons and ${ }^{3} \mathrm{He}-$ particles is shown in Figure 6. The ${ }^{3} \mathrm{He}$ signal is the largest peak in the recorded time period of $600 \mathrm{~ns}$. Also evident in the plot is the large background due to $\mathrm{x}$ rays produced by electrons that escaped the electron filter. The amplitudes of the pulses are expected to be significantly larger for D-T operation since the YAP:Ce scintillator was optimized for the higher energy $(3.5 \mathrm{MeV})$ associated $\alpha$-particles.

The same experimental setup was also used to determine the neutron source size by back-tracing the neutron trajectories from translational measurements of the neutron- ${ }^{3} \mathrm{He}$ coincidences. In this case, the plastic neutron detector was mounted on a linear stage which allowed the detector to move parallel to the direction of the deuterium beam and sweep out the coincident distribution of neutrons (Fig. 5). To account for the differences in the duration of the data acquisition time and the solid angle of the neutron detector at various positions, the coincident yield was divided by the number of identified neutron counts. The neutron source size was determined by comparing the resulting angular distribution of neutrons in coincidence with ${ }^{3} \mathrm{He}$-particles to the distribution
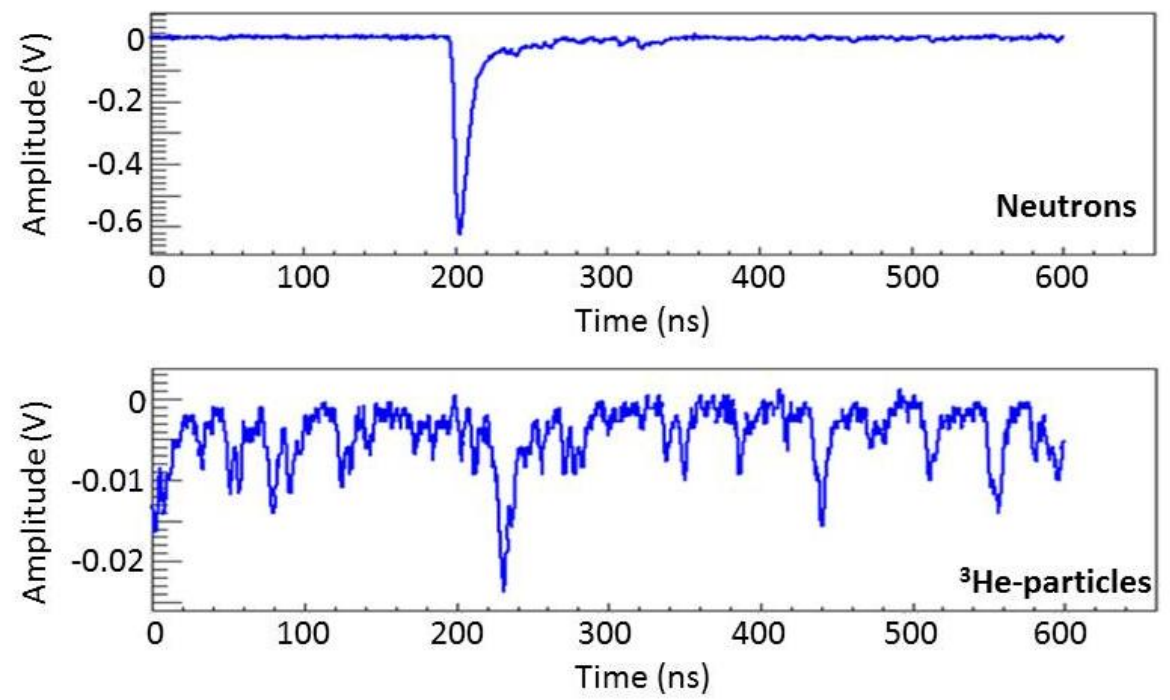

Figure 6. Digitized traces of detector signals for neutrons (top) and ${ }^{3} \mathrm{He}$-particles (bottom).

obtained with a commercial neutron generator in an identical experimental setup. ${ }^{14}$ The neutron source size in the commercial generator was fixed at 3-mm-diameter using a target mask. To compensate for different efficiencies, the coincident yield from both systems was scaled to the same value. As seen in Figure 7, the full-width-at-half-maximum of two distributions differ by approximately $60 \%$ which implies that the neutron source size no larger than 2-mm-diameter. Unfortunately, it was not possible to make a higher-fidelity measurement of the source size by this method due to the high $\mathrm{x}$-ray background in the coincidence measurements. 


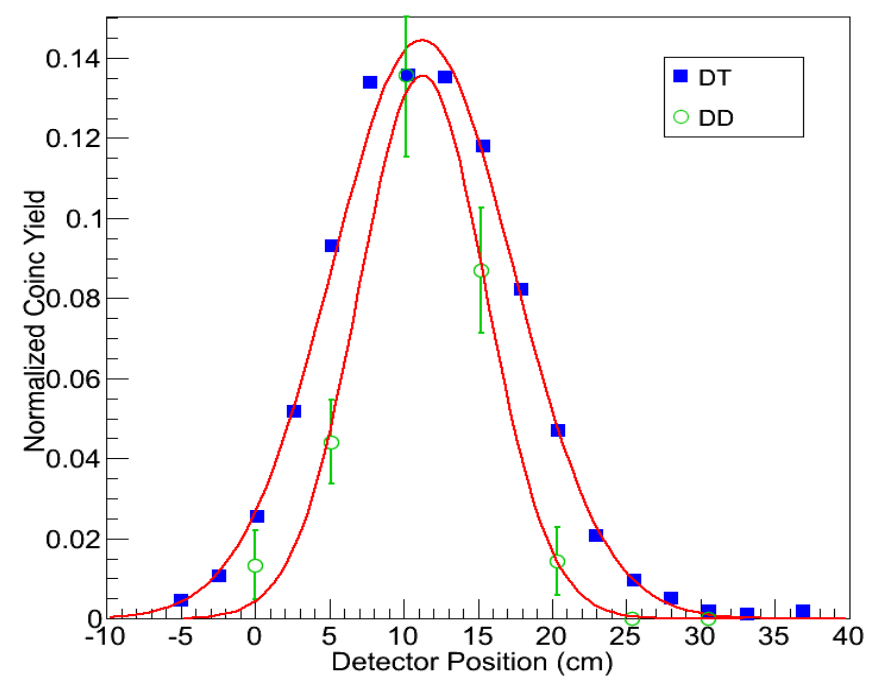

Figure 7. Angular distribution of neutrons measured in coincidence with $3.5 \mathrm{MeV}$ alphaparticles for D-T fusion in the Thermo-Fischer API-120 neutron generator (solid squares) and with $0.82 \mathrm{MeV} 3 \mathrm{He}$-particles for D-D fusion in the negative ion-driven neutron generator (open circles). The red curves are Gaussian fits to the data and are meant to guide the eye.

\section{d. Plasma Ignition}

High gas pressures (>100 mTorr) are currently needed to start the plasma in the ion source. After the plasma is ignited, the pressure is reduced and the matching network tuned to the proper levels. In a sealed system, however, the pressure inside the ion source would be difficult to adjust and an auxiliary method needs to be employed to ignite the plasma. A spark gap or small high voltage induction coil could be used for this purpose. It has previously been shown that a simple tungsten filament could emit sufficient electrons to ignite plasma with only 2 mTorr gas pressure and $1 \mathrm{~kW} \mathrm{rf-power.}{ }^{4}$ Although not implemented in the present neutron generator, an efficient means to ignite the plasma at low gas pressure would enable sealed operation.

\section{Conclusion}

A negative ion-driven associated particle neutron generator has been demonstrated under differentially pumped sealed conditions using deuterium fill gas. Negative ion current densities up to $35 \mathrm{~mA} / \mathrm{cm}^{2}$ were extracted from an efficient volume-type RF-driven plasma source without the use of cesium. The neutron generator enables reliable, high fidelity associated particle imaging by exploiting negative ions to produce high neutron yield from a "point" source, eliminate backstreaming electrons and high voltage breakdowns, and provide long operational lifetime.

\section{Acknowledgements}

The authors would like to thank Dr. S.K. Hahto for performing the ion beam optics computations. This work was supported by the DOE/NA-22 Office of Nonproliferation Research and Development. Sandia National Laboratories is a multi-program laboratory managed and operated by Sandia Corporation, a wholly owned subsidiary of Lockheed Martin Corporation, for the U.S. Department of Energy's National Nuclear Security Administration under contract DE-AC04-94AL85000. Oak Ridge National Laboratory is managed for the U.S. 
Department of Energy by UT-Batelle, LLC under Contract No. DE-AC05-00OR22725 with UTBattelle, LLC.

\section{References}

1. D.L. Chichester, M. Lemchak, J.D. Simpson, Nucl. Instr. Meth.B241 (2005) 753-758.

2. P. Le Tourneur, Proceedings of the International Topical Meeting on Nuclear Research Applications and Utilization of Accelerators, IAEA Publications, SM/EN-17 (2009); http://www-pub.iaea.org/MTCD/publications/PDF/P1433_CD/datasets/abstracts/sm_en$\underline{\text { 17.html }}$

3. V.D. Aleksandrov, E.P. Bogolubov, O.V. Bochkarev, L.A. Korytko, V.I. Nazarov, Yu.G. Polkanov, V.I. Ryzhkov, T.O. Khasaev, Applied Radiation and Isotopes 63/5-6 (2005) 537.

4. Y. Wu, J.P. Hurley, Q. Ji, J. Kwan, K.-N. Leung, IEEE Trans. Nucl. Sci. 56/3 (2009) 1306..

5. http://www.adelphitech.com/pdfs/API_generator_Flier_24-Nov-14_A.PDF

6. Y. Wu, J.P. Hurley, Q. Ji, J.W. kwan, K.-N. Leung, Rev. Sci. Instr. 81 (2010) $02 B 908$.

7. K.-N. Leung, K.W. Ehlers, C.A. Hauck, W.B. Kunkel, A.F. Lietzke, Rev. Sci. Instr. 59 (1988) 453.

8. C.W. Schmidt, Proceedings of the Linear Accelerator Conference 1990, http://accelconf.web.cern.ch/AccelConf/190/html/sessi0n.htm

9. K.-N. Leung, G.J. DeVries. W.F. DiVergilio, R.W. Hamm, C.A. Hauck, W.B. Kunkel, D.S. McDonald, M.D. Williams, Rev. Sci. Instr. 62 (1991) 100.

10. http://fti.neep.wisc.edu/presentations/alderson_aps2010.pdf

11. D.J. Malbrough, D.K. Brice, D.F. Cowgill, J.A. Borders, L.A. Shope, J.M. Harris, Nucl. Instr. Meth. B28 (1987) 459.

12. J.M. Verbeke, K.-N. Leung, J. Vujic, Applied Radiation and isotopes 53 (2000) 801.

13. S.K. Hahto, D.G. Bilbrough, R. Keller, AIP Conference Proceedings 925 (2007) 318.

14. D.L. Chichester, M. Lemchak, J.D. Simpson, Nucl. Instr. Meth. B241 (2005) 753. 